\title{
Chronic restraint stress induces hippocampal memory deficits by impairing insulin signaling
}

\author{
Hanwoong Woo ${ }^{1}$, Caroline Jeeyeon Hong ${ }^{1}$, Seonghee Jung ${ }^{1}$, Seongwon Choe ${ }^{1}$ and Seong-Woon Yu ${ }^{1,2^{*}}$
}

\begin{abstract}
Chronic stress is a psychologically significant factor that impairs learning and memory in the hippocampus. Insulin signaling is important for the development and cognitive function of the hippocampus. However, the relation between chronic stress and insulin signaling at the molecular level is poorly understood. Here, we show that chronic stress impairs insulin signaling in vitro and in vivo, and thereby induces deficits in hippocampal spatial working memory and neurobehavior. Corticosterone treatment of mouse hippocampal neurons in vitro caused neurotoxicity with an increase in the markers of autophagy but not apoptosis. Corticosterone treatment impaired insulin signaling from early time points. As an in vivo model of stress, mice were subjected to chronic restraint stress. The chronic restraint stress group showed downregulated insulin signaling and suffered deficits in spatial working memory and nesting behavior. Intranasal insulin delivery restored insulin signaling and rescued hippocampal deficits. Our data suggest that psychological stress impairs insulin signaling and results in hippocampal deficits, and these effects can be prevented by intranasal insulin delivery.
\end{abstract}

Keywords: Chronic restraint stress, Corticosterone, Insulin signaling, Intranasal insulin delivery, Nesting, Y-maze

\section{Introduction}

Stress affects various parts of our body and causes diverse physiological changes, which are manifested in symptoms such as headache, stomachache, heartburn, fatigue, overeating, or undereating. Severe and long-lasting adverse effects induced by stress include insomnia, anxiety, depression, or post-traumatic stress disorder $[1,2]$. These physiological outcomes of psychological stress are mainly caused by stress hormones [2]. Stress stimulates the hypothalamus to release corticotropin-releasing hormone $(\mathrm{CRH})$ into the portal vein. $\mathrm{CRH}$ induces anterior pituitary to release adrenocorticotrophic hormone (ACTH). ACTH affects the adrenal cortex and increases synthesis and release of corticosteroids. Corticosteroids include glucocorticoids, which regulate glucose metabolism, and mineralocorticoids, which regulate water balance and blood pressure. The major glucocorticoid is cortisol

\footnotetext{
* Correspondence: yusw@dgist.ac.kr

${ }^{1}$ Department of Brain and Cognitive Sciences, Daegu Gyeongbuk Institute of Science and Technology (DGIST), 333 Techno Jungang Daero,

Hyeonpung-Myeon, Dalseong-Gun, Daegu 42988, Republic of Korea

${ }^{2}$ Neurometabolomics Research Center, Daegu Gyeongbuk Institute of

Science and Technology (DGIST), Daegu 42988, Republic of Korea
}

in humans and corticosterone (CORT) in rodents. CORT delivered with blood causes diverse stress responses in tissues and returns to the hypothalamus and pituitary for a negative feedback on CRH and ACTH secretion [3-6].

To recapitulate psychological stress in animal models, several procedures have been developed, including unpredictable chronic stress, chronic restraint stress (CRS), and chronic administration of CORT [7-9]. CRS has been widely used as a model of chronic psychoemotional stress to induce depressive- and anxiety-like behaviors, learning and memory deficits, and hippocampal neuronal damage in mice [10, 11]. One of the most vulnerable targets of stress is hippocampus, because it abundantly expresses both glucocorticoid and mineralocorticoid receptors [12, 13]. Stress changes hippocampal neural activity and synaptic plasticity, activates hippocampal glucocorticoid receptor (GR), and decreases neuronal cell survival and neurogenesis [14-16]. Prolonged exposure to CORT also causes hippocampal neuronal damage and impairs hippocampal neurogenesis, synaptic plasticity, and learning in neuronal cultures and in mice [17-19]. 
Insulin signaling is important for the development and cognitive function of the hippocampus [20-22]. Insulin receptor (IR) is abundantly distributed in the hippocampus, and insulin binds to IR to initiate insulin/IR signaling [23]. Insulin signaling mediates neuronal development, feeding behavior, and cognitive processes [24]. Attenuated insulin production and IR activity result in learning and memory formation deficits [25], and deletion of brain IR leads to augmented anxiety, depressive-like behavior, and deficits in long-term memory [26, 27]. Blockade of IR or downstream signaling molecules such as phosphatidylinositol-3-kinase (PI3K) impairs hippocampal memory function [28, 29], whereas intrahippocampal insulin microinjection improves spatial memory [29].

Activated IR recruits and phosphorylates substrate adaptors such as the family of insulin receptor substrate (IRS) proteins. IRS-1 is a major IR substrate and a key mediator in insulin signaling. IRS-1 acts as a docking protein between the IR and intracellular signaling molecules that mediate metabolism and growth [30]. PI3K binds to activated IRS proteins and activates Akt kinase, which plays a critical role in cell survival. Akt also activates mammalian target of rapamycin (mTOR), which promotes protein synthesis and attenuates autophagy [31-33]. To target the insulin signaling pathway for the improvement of cognitive function, previous studies have tested intranasal insulin delivery to healthy subjects and showed an improvement in learning and memory in mice $[34,35]$ and in humans [36, 37]. However, only a few studies associated CORT with insulin resistance in the brain [38-40], and our understanding of the mechanisms of the effects of CORT on hippocampus, especially on insulin signaling in the hippocampus, is still lacking. Also, so far, there have been only a limited number of studies that examined the protective effects of insulin signaling potentiation against stress-induced hippocampal impairment [41].

Another unanswered question is how CORT induces neurotoxicity. One possible mechanism is that it induces apoptosis [17, 42]. However, it has not been thoroughly examined whether other modes of cell death, such as autophagic cell death, are also involved in CORT-induced neurotoxicity. Autophagy (self-eating) is an essential cellular process characterized by bulk degradation of unnecessary or dysfunctional intracellular components at basal state and under physiologically stress [43]. Double-membrane structures, autophagosomes, engulf portions of the cytosol containing intracellular components and fuse with lysosomes to form autolysosomes where cargoes are degraded by acidic lysosomal hydrolases [44]. Autophagy helps cells to cope with stress by providing metabolic intermediates and thereby contributes to cell survival $[44,45]$. On the other hand, excessive autophagy can lead to cell death [46, 47]. Autophagic cell death is defined as cell death with increased autophagic flux without the features of apoptosis or necrosis, and when cell death is prevented by suppression of autophagy [48-50].

Type II microtubule-associated protein light chain 3 (LC3-II) is a well-known biochemical marker of autophagy [51, 52]. LC3 is proteolytically cleaved immediately after its synthesis and becomes LC3-I. Cytosolic LC3-I is recruited to the autophagosome membrane, where it is conjugated with phosphatidylethanolamine and is converted into LC3-II [53]. The ubiquitin-binding protein p62 binds LC3-II and serves as a linker between LC3 and cargoes. Therefore, p62 is degraded together with the cargo molecules and a decrease in p62 level can be another marker of autophagy flux [54].

In this study, we show that CRS impairs insulin signaling in the mouse hippocampus and thereby induces deficits in hippocampal function, whereas intranasal insulin delivery prevents insulin signaling impairment and repairs hippocampal deficits. CORT treatment of primary hippocampal neurons in vitro recapitulates the impairment of insulin signaling with an increase in autophagy.

\section{Methods}

\section{Animals and CRS procedure}

All procedures that involved laboratory animals were approved by the Institutional Animal Care and Use Committee at the DGIST.

Eight-week-old male C57BL/6 N mice were housed with a 12:12 h light-dark cycle (lights on 07:00 to 19:00) with $22-24^{\circ} \mathrm{C}$ temperature, $40-60 \%$ humidity, and food and water were supplied ad libitum. Four male mice were housed in each cage until six-weeks old, and individually housed for a week and handled daily for acclimation for another week before experiments. Mice were randomly divided into the control (Ctrl) and stressed groups. Mice of the stressed group were horizontally immobilized for $6 \mathrm{~h}$ /day (from 10:00 to 16:00) in the acrylic cylindrical flat-bottom head-first restrainer $(\Phi 25 \times\{\mathrm{H}\} 90 \mathrm{~mm}$, Jeungdo Bio \& Plant Co., Seoul, Korea) for 2 weeks in their home cages. Restrainer has several slots to restrain the mouse firmly according to the size of each mouse, and suppresses the physical movement of the limbs without causing pain. After being restrained, mice were released back into their home cages immediately. Non-restraint mice (Ctrl) remained in their home cages without CRS procedure, and both Ctrl and CRS mice could not access food and water during the period of CRS exposure. The weight of the mice was measured every week before the experiment.

\section{Reagents and antibodies}

Corticosterone (Sigma-Aldrich, 27840), staurosporine (STS; Cell Signaling Technology, 9953), bafilomycin A1 
(Sigma-Aldrich, B1793), insulin-FITC (Sigma-Aldrich, I3661), methyl cellulose (Sigma-Aldrich, M0512), sodium borohydride (Sigma-Aldrich, 213462), and insulin (Roche Diagnostics, 11376497 001) were purchased from the indicated companies. Antibodies against IR subunit $\beta$ (3025), p-Akt-S473 (9271), Akt (9272), p-mTOR-S2448 (2971), mTOR (2972), and cleaved caspase-3 (C.Casp-3; 9664) were purchased form Cell Signaling Technology. Antibodies against p-IRS1-Y612 (Invitrogen, 44-816G), IRS1 (Invitrogen, PA1-1057), GR (Santa Cruz Biotechnology, SC-56851), LC3 (Novus, NB100-2220), p62 (Sigma-Aldrich, P0067) and $\beta$-actin-HRP (Santa Cruz Biotechnology, SC-47778) were purchased from the indicated companies.

\section{Hippocampal neuron culture}

Primary hippocampal neurons were obtained from C57BL/6 N mice on embryonic day 17. Briefly, hippocampi were dissociated in Hank's Balanced Salt Solution (Invitrogen, 14175-095) and maintained in a Neurobasal Medium (Gibco, 21103-049) supplemented with $100 \mathrm{U} /$ $\mathrm{ml}$ penicillin, $100 \mu \mathrm{g} / \mathrm{ml}$ streptomycin (HyClone, SV30010), 2 mM L-glutamine (Invitrogen, 25030-081), and 2\% B27 supplement (Invitrogen, 17504-044). After 3 days, one-third of the medium was replaced with medium containing cytosine $\beta$-D-arabinofuranoside (Sigma-Aldrich, C1768; final concentration, $3 \mu \mathrm{M}$ ). After 4 days, one-third of the medium was replaced with fresh medium without cytosine $\beta$-D-arabinofuranoside and cultures were used for experiments on day 11 or 12 days.

\section{Cell death assay}

Cell death was assessed by dual staining of cells with the membrane-permeable dye Hoechst 33342 (Invitrogen, H1399) to stain all cells and with the membrane-impermeable dye propidium iodide (PI; Sigma-Aldrich, P4170) to stain dead cells. The percentage of cell death (\%) was calculated by dividing the number of red-positive (PI) cells by that of blue-positive (Hoechst 33342) cells.

\section{Confirmation of intranasal delivery of insulin into the hippocampus using insulin-FITC}

Insulin-FITC reconstituted in $0.01 \mathrm{~N} \mathrm{HCl}$ was diluted in $0.9 \%$ saline with $0.001 \%$ methyl cellulose and was delivered twice into both nares: the first dose $6 \mathrm{~h}$ and the second dose at $1 \mathrm{~h}$ before sacrifice $(2.5 \mu \mathrm{g} / 24 \mu \mathrm{l}$ each). Control animals received $0.9 \%$ saline with $0.001 \%$ methyl cellulose.

\section{Intranasal delivery of insulin}

Mice were hand-restrained in a supine position with the neck in extension and were not anesthetized. A total of $100 \mu \mathrm{g}$ of insulin in $20 \mu \mathrm{l}$ of vehicle $(0.9 \%$ saline with
$0.001 \%$ methyl cellulose) or the same volume of vehicle alone was delivered daily into both nares simultaneously 30 min before restraint stress for 7 days.

\section{Nest-building assay}

Two nestlets were added in each cage at 16:00 on the 13th day after restraint stress and scores were measured on the 14th day (09:50) before the start of restraint stress.

\section{Y-maze assay}

Y-maze test was used to evaluate spatial working memory of the mice. The test was performed in a Y-shaped maze with three white opaque plastic arms. Mice were placed into the center of the maze and were allowed to explore the three arms freely for $6 \mathrm{~min}$. Each experiment was video recorded with EthoVision software (Noldus, EthoVision XT 11.5). After exploration, alternation (\%) was calculated as the number of three consecutive arm entries divided by the number of possible alternations (total arm entries minus two).

\section{CORT level measurement}

Mouse blood serum samples were acquired on the 14th day 30 min after the end of stress from the submandibular vein using animal lancets. Blood CORT levels were measured using an ELISA kit (Enzo Life Sciences, ADI-900-097).

\section{Preparation of hippocampal lysates}

Mice were deeply anesthetized by injecting Zoletil (40 mg/kg) and Rompun (5 mg/kg) and sacrificed by decapitation. Brains were removed, dissected, and hippocampi were isolated on ice and stored in liquid nitrogen. Hippocampi were lysed in radioimmunoprecipitation assay (RIPA) buffer (Sigma-Aldrich, R0278) containing $1 \mathrm{mM}$ phenylmethanesulfonyl fluoride (PMSF; Sigma-Aldrich, 78830), $1 \mathrm{mM}$ dithiothreitol (DTT; Sigma-Aldrich, D9779), 1× protease (Thermo Scientific, 87786) and phosphatase (Thermo Scientific, 78420) inhibitor cocktails by using a $1 \mathrm{ml}$ syringe with 10 strokes, and were further disrupted with Bioruptor (Cosmobio, KRB-01) for $20 \mathrm{~min}$. Following centrifugation $(12,000 \times g, 10 \mathrm{~min})$, the supernatants were harvested and the protein concentrations were measured using a BCA protein assay reagent (Thermo Scientific, 23224).

\section{Western blotting analysis}

Harvested hippocampal neurons were lysed in RIPA buffer containing $1 \mathrm{mM}$ PMSF, $1 \mathrm{mM}$ DTT and $1 \times$ protease and phosphatase inhibitor cocktails for $20 \mathrm{~min}$ on ice. Following centrifugation $(12,000 \times g, 10 \mathrm{~min})$, protein concentrations were measured using the BCA protein assay reagent. Typically, $30 \mu \mathrm{g}$ of total protein of hippocampal tissue or 
neuron lysates was loaded per well. Proteins were electro-transferred to polyvinylidene fluoride membranes (Millipore, IPVH00010) in a semidry electrophoretic transfer cell (Bio-Rad). The membranes were incubated overnight with primary antibodies. After washing, the membranes were incubated for $1 \mathrm{~h}$ at room temperature with peroxidase-conjugated secondary antibodies. After washing, the membranes were processed for analysis using a chemiluminescence detection kit (Thermo Scientific, 34080).

\section{Immunocytochemistry}

Mouse hippocampal neurons were fixed with $4 \%$ paraformaldehyde (PFA) and permeabilized with $0.1 \%$ Triton $\mathrm{X}-100$ in phosphate buffered saline (PBS) containing $0.5 \%$ bovine serum albumin (BSA; Affymetrix/USB, 10857). Following blocking with $0.5 \%$ BSA, the samples were incubated overnight with cleaved caspase- 3 antibody at 1:1000 dilutions and then with an anti-rabbit Alexa 488 secondary antibody (Jackson ImmunoResearch, 711-545-152). Samples were stained with Hoechst 33342 and visualized under a confocal laser scanning microscope (Carl Zeiss, LSM 700); images were analyzed using ZEN12 software (Carl Zeiss).

\section{Tissue histology}

Mice were anesthetized by Zoletil and Rompun injection and perfused with PBS, followed by $4 \%$ PFA. Brains were then removed, post-fixed in 4\% PFA for $12 \mathrm{~h}$ and cryoprotected in $30 \%$ sucrose until they sank to the bottom of the tube. For fluorescence microscopy, brains were frozen in optimal cutting temperature compound and cryosectioned coronally with a $30 \mu \mathrm{m}$ thickness. Brain section coordinates were based on the mouse brain atlas [55]. The sections were free-floated in PBS, blocked with $0.1 \%$ sodium borohydride in PBS to reduce background autofluorescence, counter stained with Hoechst 33342, and mounted. FITC-tagged insulin was visualized by a slide scanner (Zeiss, Axio Scan.Z1) and images were analyzed using ZEN12 software.

\section{Statistical analysis}

All data were obtained from at least three independent experiments and are presented as mean values \pm standard error of the mean (SEM). Statistical significance was determined using one-way analysis of variance (ANOVA) and Tukey's post-test. Unpaired t test, nonparametric Mann-Whitney U test, or two-way ANOVA and Bonferroni post-test were also used in experiments, as indicated in the figure legends. Differences were considered statistically significant at $p<0.05$.

\section{Results}

CORT is neurotoxic and induces autophagy features in mouse hippocampal neurons in vitro

Chronic psychological stress or long-term application of CORT results in time-dependent hippocampal neuronal damage [17-19, 38, 42]. However, the underlying mechanisms of stress-induced hippocampal neuronal damage are still not well understood. To examine the neurotoxicity of CORT, we treated mouse hippocampal neurons in vitro with a wide range of CORT doses and found that 100 or $200 \mu \mathrm{M}$ CORT significantly increased hippocampal neuronal cell death (Fig. 1a). Because there were reports that glucocorticoids induced neuronal apoptosis $[17,42]$, we examined whether the CORT-induced neurotoxicity was apoptotic or not. We used STS, a widely used apoptosis inducer, as a positive control of apoptosis [56]. C.Casp-3 is active form of caspase-3 and is the best-known marker of apoptosis [57]. Of interest, STS but not $200 \mu \mathrm{M}$ CORT induced robust C.Casp-3 immunoreactivity (Fig. 1b). Moreover, nuclear condensation, another well-known characteristic of apoptosis, was observed in STS-treated but not in CORT-treated cells (Fig. 1b, arrow). Immunoblot analysis showed that treatment with STS but not with $200 \mu \mathrm{M}$ CORT induced C.Casp-3 (Fig. 1c). Since apoptotic markers were not detected after CORT treatment, we wondered whether another mode of programmed cell death, such as autophagic cell death $[44,48,58]$, is involved. CORT increased the level of LC3-II (Fig. 1d, e). Bafilomycin A1 (Baf.A1) is a well-known inhibitor of the late phase of autophagy and is used to prevent maturation of autophagic vacuoles by inhibiting fusion between autophagosomes and lysosomes [59]. Although an increase in LC3-II level is typically regarded as an indicator of increased autophagy flux, impaired autophagy flux and blockage of autophagic degradation can also result in LC3-II accumulation level, because LC3-II itself is degraded by autophagy. These possibilities can be distinguished by blocking the late phase of autophagy flux. Further accumulation of LC3-II after treatment Baf.A1 would indicates an increase in autophagic flux, not the suppression of autophagic degradation $[52,56]$. On the other hand, if the LC3-II level is not significantly affected by Baf.A1, it may suggest impaired autophagy flux. Since p62 is also degraded in autolysosomes, blocking of autophagosome fusion with lysosomes or inhibition of lysosomal hydrolases can cause p62 accumulation under the conditions of high autophagy flux. Baf.A1 treatment of control cells for $2 \mathrm{~h}$ led to accumulation of p62 and LC3-II (Fig. 1d, e). CORT treatment increased LC3-II and decreased p62, and the CORT+Baf.A1 group accumulated p62 together with a higher increase in LC3-II than in the CORT-alone group (Fig. 1d, e). These data suggest that CORT does not induce apoptosis, but enhances autophagy 


\section{a}

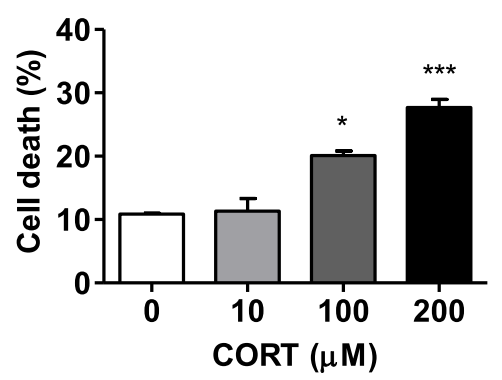

C

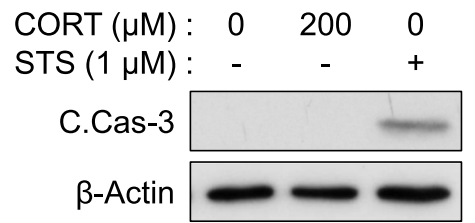

b

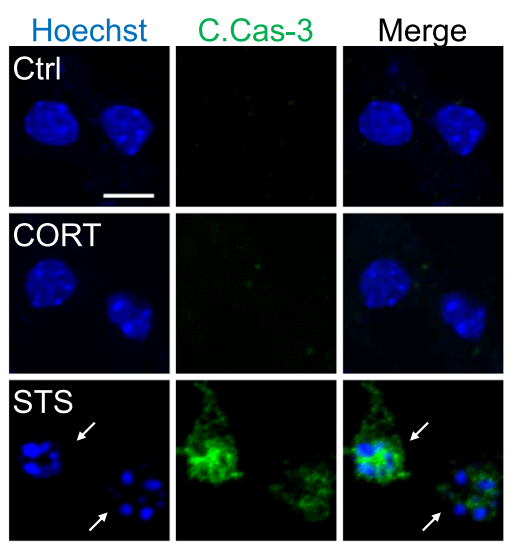

d

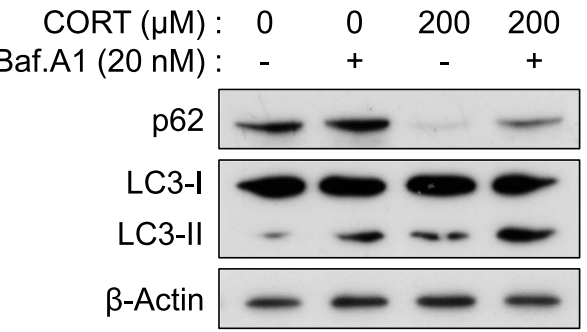

e
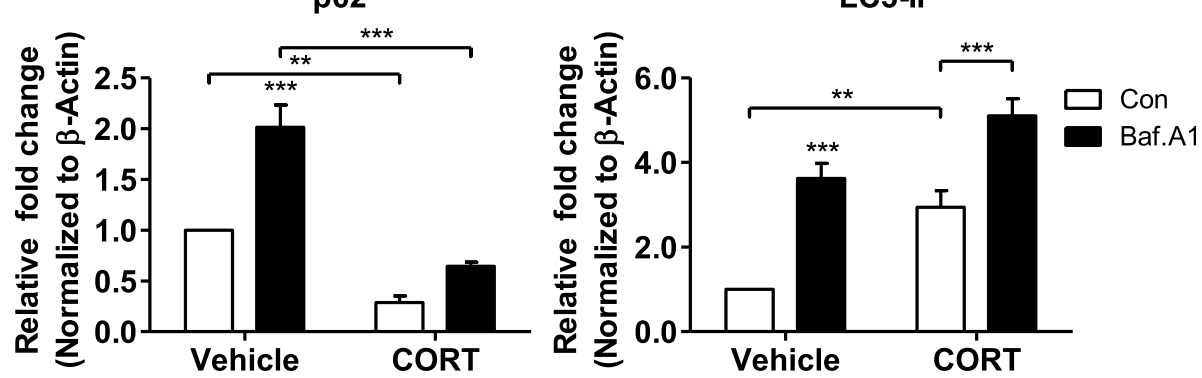

Fig. 1 Neurotoxicity of CORT in primary mouse hippocampal neurons. a Dose dependence analyzed by one-way ANOVA ( $n=3$ ). b Immunostaining for cleaved caspase-3 (C.Casp-3) after treatment with CORT for $24 \mathrm{~h}$ or staurosporine (STS) for 6 h. Arrows indicates apoptotic, condensed nucleus. Scale bar, $10 \mu \mathrm{m}$. c Western blotting analysis of C.Casp-3 after treatment with CORT for 24 h or STS for 6 h. d Western blotting analysis of LC3-II after CORT treatment for $24 \mathrm{~h}$. Bafilomycin A1 (Baf.A1; $20 \mathrm{nM}$ ) was added 2 h before sampling. e Quantification of p62 and LC3-II analyzed by two-way ANOVA $(n=8)$. Data are mean \pm SEM. ${ }^{*} p<0.05,{ }^{* *} p<0.01,{ }^{* * *} p<0.001$

flux in mouse hippocampal neurons, suggesting the involvement of autophagic cell death in CORT-induced neurotoxicity.

\section{CORT impairs insulin signaling in mouse hippocampal neurons in vitro}

Next, we examined whether CORT affects insulin signaling, since insulin signaling is closely related to autophagy and is also important for hippocampal development and function, and for survival of hippocampal neural stem cells and neurons [47, 48, 60]. Treatment of mouse hippocampal neurons with $200 \mu \mathrm{M}$ CORT increased the expression of GR, a positive marker of CORT action (Fig. 2a, b). CORT treatment downregulated phosphorylation of IRS1 on Y608 (Y612 in humans) without changing the total IRS1 level (Fig. 2a, b). The phosphorylation of Akt on S473 and mTOR on S2448 was also decreased after CORT treatment (Fig. 2a, b). Intriguingly, the expression of IR subunit $\beta$ (IR $\beta)$ was increased by CORT treatment (Fig. 2a, b), which might be a compensation to overcome the impaired insulin signaling [48]. Taken together, these results suggest that CORT impairs insulin signaling (the IRS1-Akt-mTOR pathway) in mouse hippocampal neurons. 

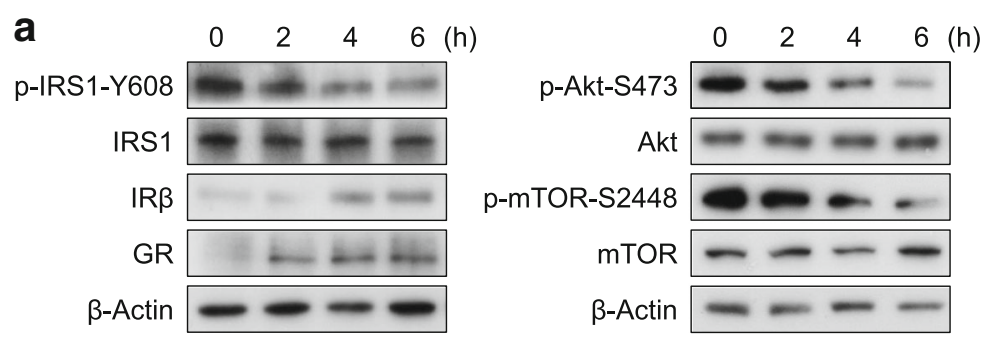

b
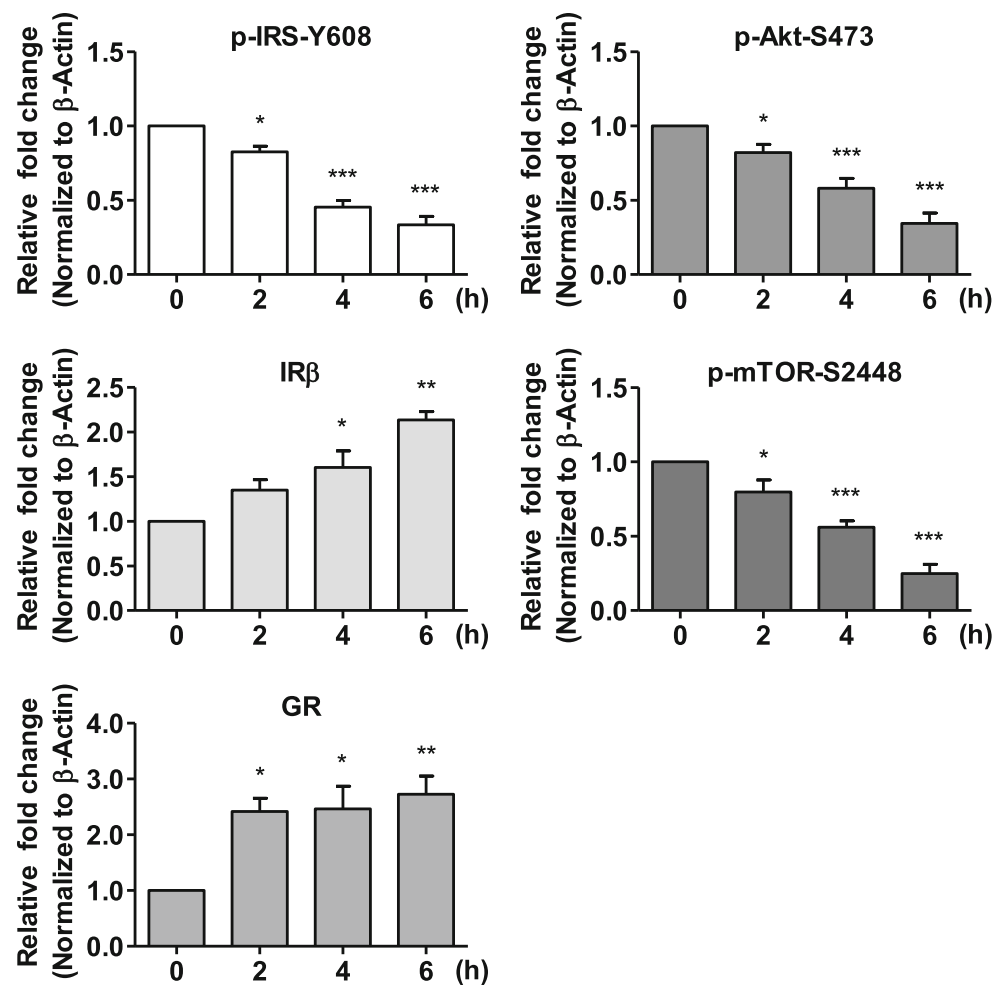

Fig. 2 Impairment of the insulin signaling pathway following CORT treatment in primary mouse hippocampal neurons. a Western blotting analysis of insulin signaling molecules. b Quantification of insulin signaling molecules analyzed by one-way ANOVA ( $n=4)$. Blots are representative of four experiments with similar results. Data are mean $\pm \mathrm{SEM}$. ${ }^{*} p<0.05,{ }^{* *} p<0.01,{ }^{* * *} p<0.001$

CRS impairs insulin signaling in the mouse hippocampus in vivo

Next, we wondered whether psychological stress, such as CRS, which involves high levels of corticoid hormones, affects insulin signaling in vivo. To generate CRS, we used a flat bottom restrainer for $6 \mathrm{~h} /$ day for 2 weeks (Fig. 3a). First, we measured stress responses during and after CRS. Whereas the initial body weight did not differ between the CRS and Ctrl groups, CRS significantly inhibited body weight gain compared to Ctrl (Fig. 3b) and significantly increased the circulating blood CORT level after 2 weeks (Fig. 3c). We used nesting behavior as another index of neuronal stress. Nesting behavior depends on normal hippocampal function and is impaired by hippocampal lesions [61]. Nesting behavior was measured by scoring the quality of the nest with a 5-point rating scales. Rating scale 1 means that nestlets are not touched, and 5 means a nearly perfect nest with a crater and higher walls. Unlike the Ctrl group, the CRS group had difficulties in building a nest with nestlets (Fig. 3d, e).

To determine whether CRS influences insulin signaling in the hippocampus, we measured the expression and phosphorylation of relevant signaling proteins by immunoblotting (Fig. 3f, g). The expression of GR was increased in the hippocampi of the CRS group, which is likely a response to an increased blood CORT level. Similar to hippocampal cultures treated with CORT, CRS increased the expression of IR $\beta$. Interestingly, the levels of p-IRS1-Y608, p-Akt-S473 and p-mTOR-S2448 were notably decreased in the CRS group. In summary, these data demonstrate successful induction of stress 
a
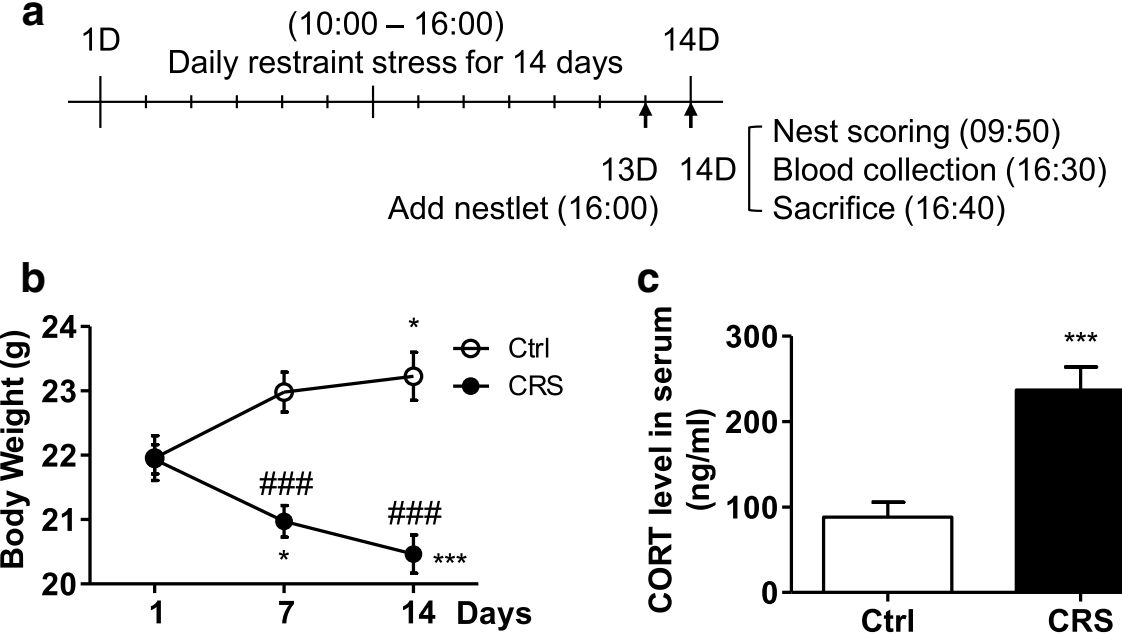

d

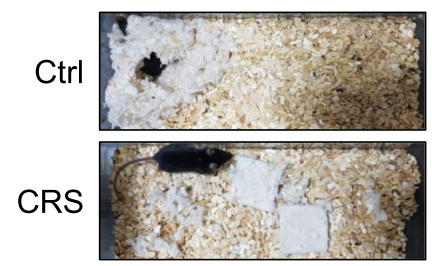

f

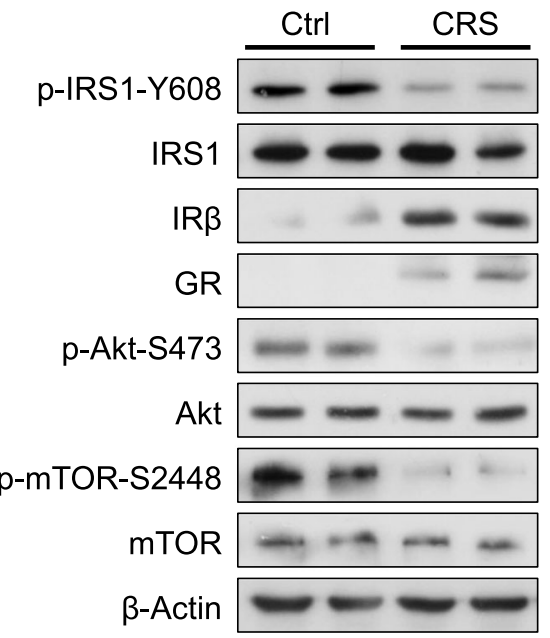

C

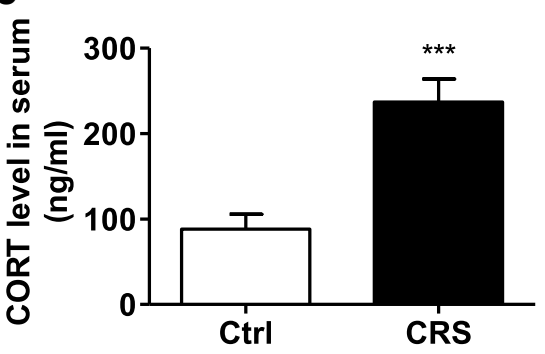

e

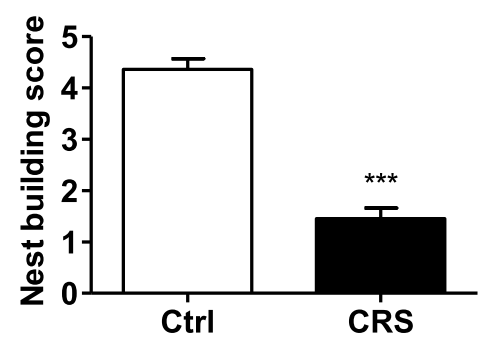

g

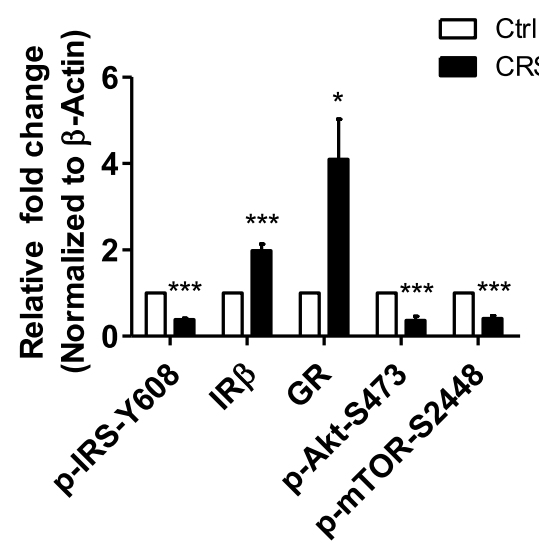

Fig. 3 Impairment of insulin signaling pathways and nesting behavior in mice subjected to chronic restraint stress (CRS). a Experimental scheme. b Body weight analyzed by two-way ANOVA ( $n=11$ per group). ${ }^{*} p<0.05,{ }^{* * *} p<0.001$ (difference between days in the same groups); \#\#\#p 0.001 (difference between Ctrl and CRS groups on the same days). $\mathbf{c}$ Concentration of blood CORT analyzed by unpaired t test $(n=6)$. $\mathbf{d}$ Nest building behavior assay. Representative results are shown. e Nest building scores analyzed by nonparametric Mann-Whitney $U$ test ( $n=11$ per group). f Western blotting analysis of the insulin signaling pathway in the mouse hippocampus ( $n=7$ per group). $\mathbf{g}$ Quantification of insulin signaling molecules analyzed by unpaired t test. Data are mean \pm SEM. ${ }^{*} p<0.05,{ }^{* * *} p<0.001$

and subsequent impairment of insulin signaling in the mouse hippocampus following 2 weeks of CRS.

\section{Intranasal insulin delivery reduces CRS-induced memory} impairment in mice

To test whether down-regulated insulin signaling can be recovered by insulin delivery to the hippocampus, we delivered insulin intranasally. First, to confirm that insulin arrived into the hippocampus, we tagged insulin with FITC and tracked it by histology image analysis after intranasal delivery (Fig. 4a). Administration of insulin-FITC but not saline resulted in green fluorescence in the dentate gyrus areas of the hippocampus $6 \mathrm{~h}$ after administration (Fig. 4b). Administration of insulin-FITC in CRS mice 


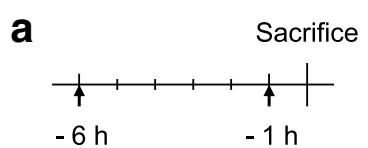

Intranasal insulin-FITC delivery

$(2.5 \mu \mathrm{g} / 24 \mu \mathrm{l}$ each)
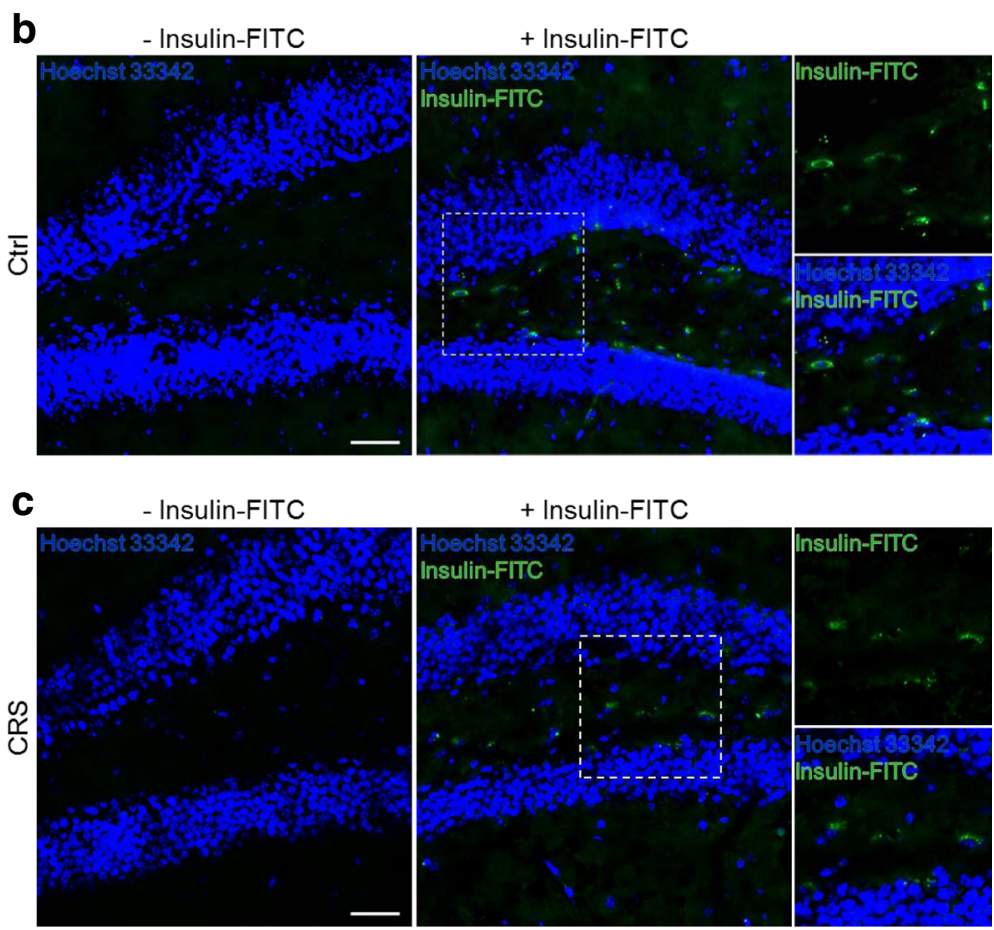

Fig. 4 Detection of insulin-FITC after intranasal delivery. a Experimental scheme. b Representative images of hippocampal tissues without and with insulin-FITC in Ctrl mice ( $n=3$ per group). Anterior-posterior coordinates of images according to mouse brain atlas are Bregma $-1.90 \mathrm{~mm}$ (- Insulin-FITC) and $-2.14 \mathrm{~mm}$ (+ Insulin-FITC) respectively. Scale bar, $50 \mu \mathrm{m}$. c Representative images of hippocampal tissues without and with insulin-FITC in CRS mice ( $n=3$ per group). Anterior-posterior coordinates of images according to mouse brain atlas are Bregma $-1.96 \mathrm{~mm}$ (- Insulin-FITC) and $-2.26 \mathrm{~mm}$ (+ Insulin-FITC) respectively. Scale bar, $50 \mu \mathrm{m}$

showed similar green fluorescence distribution (Fig. 4c). These data validated successful delivery of insulin into the hippocampus in both Ctrl and CRS mice. Next, we divided mice into 4 groups: Ctrl with saline (Ctrl/Saline), CRS with saline (CRS/Saline), Ctrl with insulin (Ctrl/Insulin), and CRS with insulin (CRS/Insulin). Insulin or saline was intranasally delivered for 7 days during the 2nd week of CRS (Fig. 5a). Both Ctrl/Saline and Ctrl/Insulin groups showed an increased in body weight, while the CRS/Saline group showed significantly reduced body weight (Fig. 5b). Interestingly, the CRS/Insulin group also showed reduced body weight after 1-week-CRS, but the reduction was reversed after intranasal administration of insulin (Fig. 5b). This shows a potential beneficial effect of intranasal insulin delivery for the functional and metabolic recovery of stressed hippocampus. Blood CORT level and nest-building behavior were also measured after experiments. Interestingly, intranasal administration of insulin did not alter the level of CORT increased by CRS (Fig. 5c).
This data suggest that the neuroprotective activity of insulin is by suppressing autophagy in the hippocampus against the detrimental effects of CORT, but not by alteration of the Hypothalamic-Pituitary-Adrenal axis. The CRS/Insulin group also regained the ability to build a nest compared to the CRS/Saline group (Fig. 5d, e).

We next measured the function of hippocampal spatial working memory by using the Y-maze test. Rodents typically prefer to explore a new arm of a maze instead of going to the previously visited arm. The Y-maze test measures the willingness of mice to explore new environments by counting the number of arm entries and calculating the percentage of alternation, which is related to the hippocampal spatial working memory. Normal mice show a high percentage of alternation. Thus, this test can easily quantify the cognitive deficits in mice $[62,63]$. The number of total arm entries was similar in all four groups of mice, showing intact general locomotor activity (Fig. 5f, top). However, the CRS/Saline group 
a

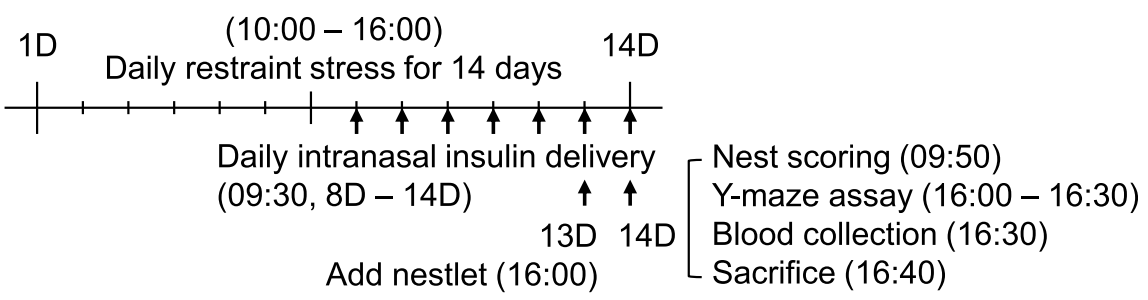

b

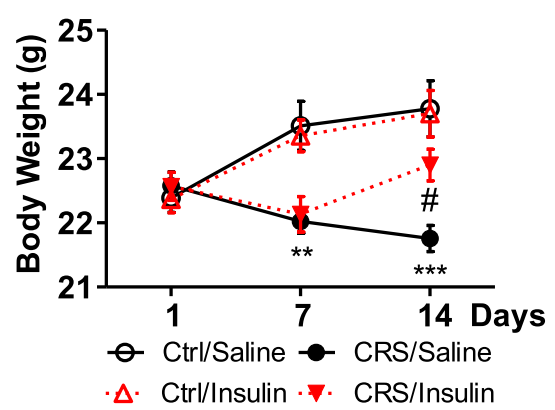

d
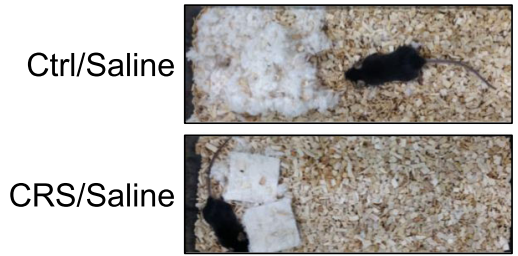

Ctrl/Insulin
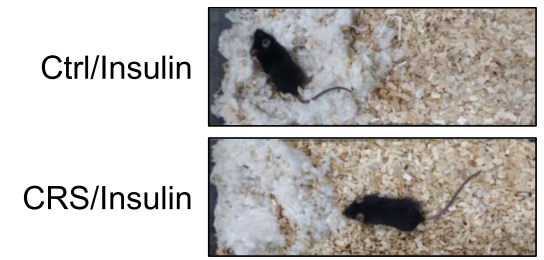

$\mathbf{e}$

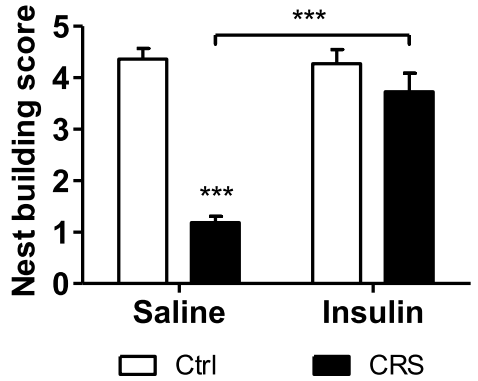

C $\frac{E}{2}$

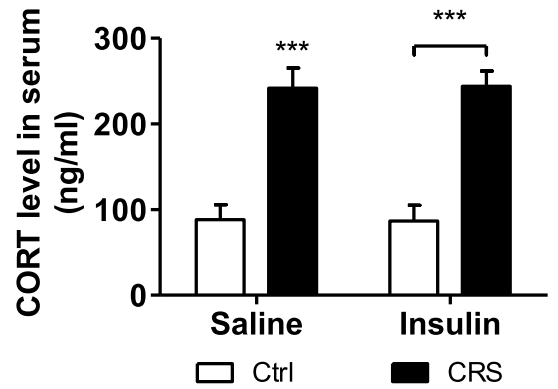

$\mathbf{f}$
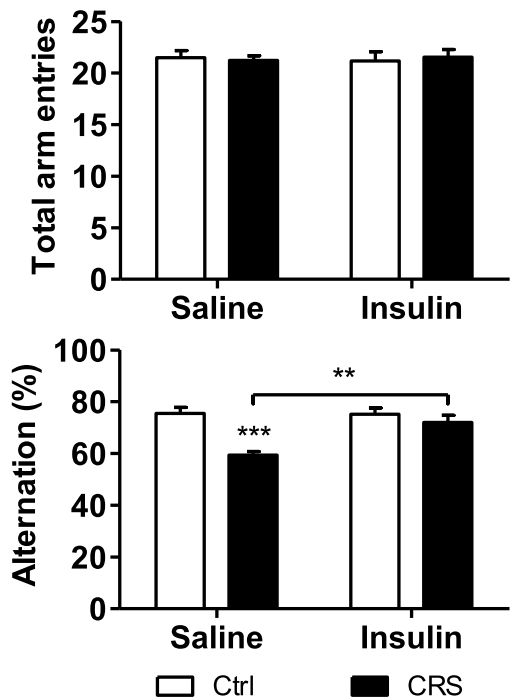

Fig. 5 Mitigation of CRS-induced hippocampal damage by intranasal insulin delivery. a Experimental scheme. b Body weight analyzed by two-way repeated ANOVA ( $n=9$ per group). ${ }^{* *} p<0.01,{ }^{* * *} p<0.001$ (difference between Ctrl/Saline and CRS/Saline groups on the same days); $\# p<0.05$ (difference between CRS/Saline and CRS/Insulin groups on the same days). c Concentration of blood CORT analyzed by two-way ANOVA (Ctrl/Saline, $n=6 ;$ Ctrl/Insulin, $n=6 ; C R S / S a l i n e, n=7 ; C R S / I n s u l i n, n=7)$. $\mathbf{d}$ Nest building behavior assay. Representative results are shown. e Nesting building scores analyzed by two-way ANOVA (bottom) $(n=11$ per group). $\mathbf{f}$ Y-maze test analyzed by two-way ANOVA (Ctrl/Saline, $n=6$; Ctrl/Insulin, $n=6 ;$ CRS/Saline, $n=9 ;$ CRS/Insulin, $n=9$ ). Data are mean \pm SEM. ${ }^{* *} p<0.01,{ }^{* * *} p<0.001$. Ctrl/saline, control group with saline; CRS/saline, CRS group with saline; Ctrl/insulin, control group with insulin administration; CRS/insulin, CRS group with insulin administration

showed a significantly reduced percentage of alternation compared to the Ctrl/Saline group, while the CRS/Insulin group recovered it to a level similar to those of Ctrl groups (Fig. 5f, bottom). To confirm biochemically whether intranasal insulin delivery recovered insulin signaling impaired by CRS, we measured the expression and phosphorylation levels of the relevant signaling proteins in the hippocampus by immunoblotting analysis (Fig. 6a). The expression levels of GR and IR $\beta$ were increased only in the CRS/Saline group (Fig. 6b, left). Interestingly, the levels of p-IRS1-Y608, p-Akt-S473, and p-mTOR-S2448 were reduced in the CRS/Saline groups but recovered in 
a
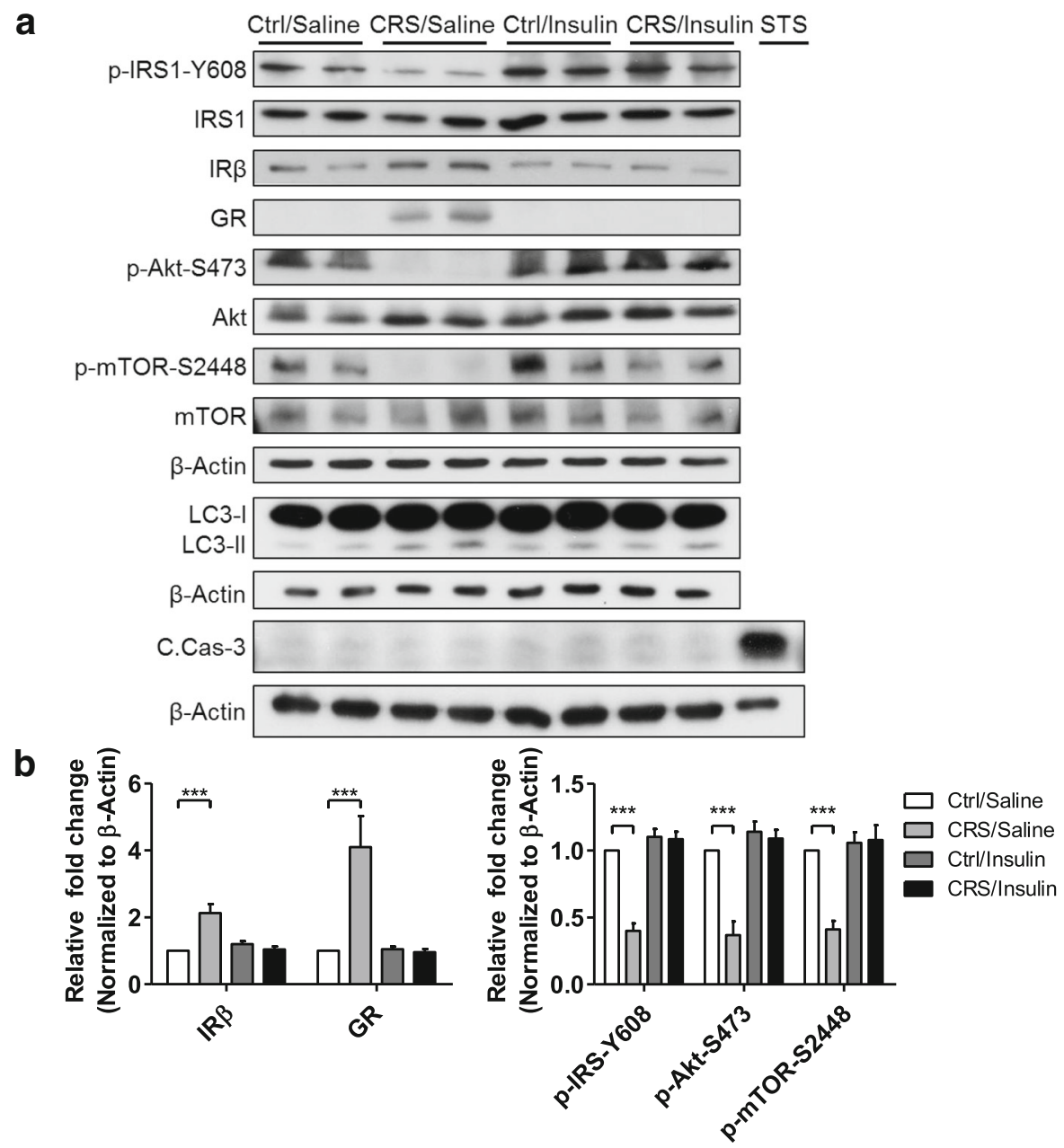

C
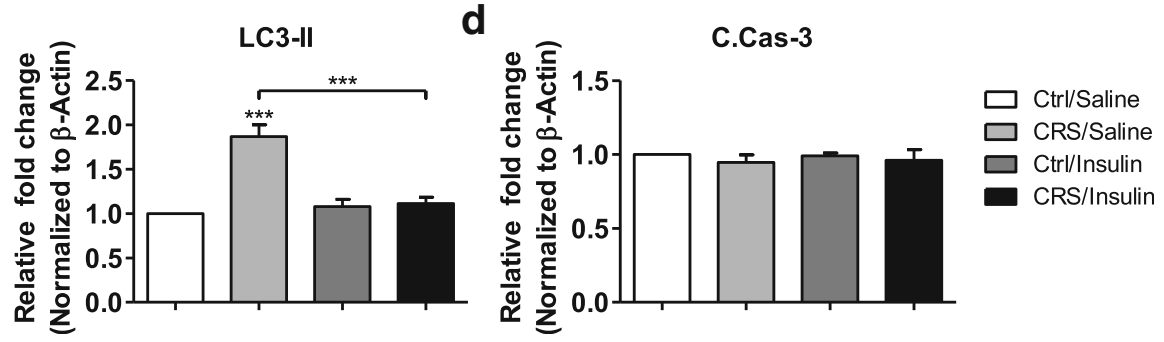

Fig. 6 Prevention of CRS-induced hippocampal insulin signaling impairment and autophagy by intranasal insulin delivery. a Western blotting analysis of the insulin signaling pathway and the characteristics of cell death in the mouse hippocampus. $\mathbf{b}$ Quantification of each insulin signaling molecules analyzed by two-way ANOVA (IRB, $n=9 ;$ GR, $n=7$; p-IRS-Y608, $n=13$; p-Akt-S473 $n=10$; p-mTOR-S2448, $n=7$ ). c Quantification of LC3-II analyzed by two-way ANOVA ( $n=5$ per group). d Quantification of C.Casp-3 analyzed by two-way ANOVA ( $n=4$ per group). Data are mean \pm SEM. ${ }^{* * *} p<0.001$

the CRS/Insulin groups (Fig. 6b, right). Altogether, these data suggest that intranasal insulin delivery relieved stress in the hippocampus.

Previous study reveals that insulin withdrawal causes a caspase-independent autophagic cell death in adult hippocampal neural stem cells [48], and we confirmed CORT impairs insulin signaling and induces autophagy features in mouse hippocampal neurons in Figs. 1 and 2.
We next examined whether CRS-induced impairment of insulin signaling induced the feature of apoptosis or autophagy. LC3-II levels in the hippocampal lysates were increased by CRS, which were suppressed by intranasal insulin administration (Fig. 6c). However, the level of C.Casp-3 was not significantly different between Ctrl and CRS mice hippocampus regardless of intranasal administration of insulin (Fig. 6d). STS-treated in vitro 
hippocampal neuronal lysate was loaded as a positive marker of C.Casp-3 (Fig. 6a, last lane).

In summary, our data demonstrate that, although psychological stress impairs hippocampal functions and insulin signaling, intranasal insulin delivery can efficiently prevent CRS-induced insulin signaling impairment and rescue deficits in hippocampus-dependent neurobehavior.

\section{Discussion}

Here, we demonstrated that CORT induces cell death in mouse hippocampal neurons with the characteristics of autophagy rather than apoptosis. CORT was neurotoxic and impaired insulin signaling; namely, it reduced the levels of p-IRS1-Y608, p-Akt-S473, and p-mTOR-S2448 and increased the expression of IR $\beta$. We also observed that CRS impaired insulin signaling in the mouse hippocampus, as revealed by reduced levels of p-IRS1-Y608, p-Akt-S473, and p-mTOR-S2448. Of interest, intranasal insulin delivery to CRS mice restored impaired insulin signaling and rescued hippocampal cognitive deficits, indicating that dysregulated insulin signaling underlies defective hippocampal function following psychological stress.

It remains elusive how CORT causes neuronal damage. CORT reportedly induces apoptosis in primary hippocampal neurons [42, 64, 65], although the incidence of apoptosis in rodent chronic stress models is rare despite hippocampal volume shrinkage and neuronal loss after physiological stress [66-68]. Interestingly, we observed an increase in autophagy flux, but failed to detect activation of caspase- 3 in CORT-treated neurons. Moreover, a pan-caspase inhibitor, Z-VAD-FMK, did not prevent cell death after CORT treatment (data not shown). Further studies using genetic knockout mouse models with neuron-specific deletion of autophagy genes will be required to prove the autophagic nature of CORT- and CRS-induced neurotoxicity.

The molecular mechanisms underlying the effects of CORT on insulin signaling in the hippocampus are poorly understood [38-40]. In this study, we revealed that CORT impaired insulin signaling in the hippocampus, which notably reduced the phosphorylation of IRS1 on Y608. This residue is considered as a PI3K-binding site [69]. Therefore, the Y608 residue of IRS1 may be a primary target of CORT in the insulin signaling cascade.

It is an interesting question why insulin signaling is impaired in the hippocampus under psychological stress. Acute psychological stress using inescapable foot shock has no effect on the IR-IRS1-Akt pathway in the brain [70]. Therefore, different models of stress as well as duration and strength of stress could yield different outcomes in terms of hippocampal insulin signaling.

Moosavi et al. demonstrated that CRS impaired spatial performance of mice in the Morris water maze and a high dose of insulin microinjected into the hippocampus prevented this deficit [41]. However, intrahippocampal insulin microinjection is invasive and may result in infection and secondary tissue damage. Here, we used a less aversive and non-invasive intranasal insulin delivery procedure and confirmed successful delivery of insulin to the hippocampus within a few hours and enhanced insulin signaling in the hippocampus. Intranasal insulin administration also effectively recovered hippocampal functions, including spatial working memory and nesting behavior. This suggests that intranasal insulin administration can be a simple and convenient strategy for the therapy of neurodegenerative disorders such as Alzheimer's disease which are also accompanied by the impairment of insulin signaling. Furthermore, intranasal insulin delivery in CRS-treated mice reduced the loss of body weight, suggesting additional benefits for other hippocampal functions, although this may be an indirect effect. On the basis of these results, we suggest that intranasal insulin delivery to the hippocampus can be applied to various neuropsychiatric disorders and neurodegenerative conditions, as it is effective for stress relief and neuroprotection, and is less aversive and easy to apply, although there is a possibility that insulin may act in other brain regions as well as hippocampus.

Although we have demonstrated that CORT impairs insulin signaling in hippocampal neurons in vitro, we have not confirmed whether CRS impairs insulin signaling in hippocampal neurons in vivo, as hippocampal tissue contains many types of cells including neural stem cells, neurons, and glia. Hence, it needs to be evaluated in which types of hippocampal cells insulin signaling is affected by CRS. Other unanswered questions include whether autophagy occurs in hippocampal neurons in vivo or how IRS1 phosphorylation on -Y608 is regulated. Nevertheless, our results indicate the potential involvement of autophagy in psychological stress-induced neuropathology and the effectiveness of insulin for neuroprotection and cure of hippocampal cognitive dysfunction.

\section{Abbreviations \\ ACTH: Adrenocorticotropic hormone; Baf.A1: Bafilomycin A1; BSA: Bovine serum albumin; C.Casp-3: Cleaved caspase-3; CORT: Corticosterone; $\mathrm{CRH}$ : Corticotropin- releasing hormone; CRS: Chronic restraint stress; Ctrl: Control; DTT: Dithiothreitol; GR: Glucocorticoid receptor; IR: Insulin receptor; IRS: Insulin receptor substrate; IRB: Insulin receptor subunit $\beta$; LC3: Microtubule-associated protein light chain 3; mTOR: Mammalian target of rapamycin; PBS: Phosphate buffered saline; PFA: Paraformaldehyde; PI: Propidium iodide; PI3K: Phosphatidylinositol-3- kinases; PMSF: Phenylmethanesulfonyl fluoride; RIPA \\ buffer: Radioimmunoprecipitation assay buffer; SEM: Standard error of mean; STS: Staurosporine}

\section{Funding}

This work was supported by the National Research Foundation of Korea (NRF) grant (2017R1A2B4004289), the Korean Brain Research Institute basic research program (18-BR-04), and the DGIST Convergence Science Center Program (18-BD-04) of the Ministry of Science and ICT of Korea. 


\section{Availability of data and materials}

All data generated or analyzed during this study are included in this article.

\section{Authors' contributions}

HW, S-WY designed the research; HW, CJH, SJ, SC performed the research; HW analyzed the data; and HW, CJH, SJ, SC, S-WY wrote the paper. All authors read and approved the final manuscript.

\section{Ethics approval}

All the animal experiments were approved by the Institutional Animal Care and Use Committee at DGIST. Consent to participate is not applicable.

\section{Consent for publication}

Not applicable.

\section{Competing interests}

The authors declare that they have no competing interests.

\section{Publisher's Note}

Springer Nature remains neutral with regard to jurisdictional claims in published maps and institutional affiliations.

\section{Received: 10 February 2018 Accepted: 22 June 2018} Published online: 03 July 2018

\section{References}

1. Schacter DL, Gilbert DT, Wegner DM: Psychology 2nd edn. New York, NY: Worth Publishers; 2011.

2. de Kloet ER, Joels M, Holsboer F. Stress and the brain: from adaptation to disease. Nat Rev Neurosci. 2005;6:463-75.

3. Nestler EJ, Hyman SE, Malenka RC: Mol Neuropharmacol : a foundation for clinical Neuroscience 2nd edn. New York: McGraw-Hill Medical; 2009.

4. Aguilera G. HPA axis responsiveness to stress: implications for healthy aging. Exp Gerontol. 2011;46:90-5.

5. Andrews RC, Walker BR. Glucocorticoids and insulin resistance: old hormones, new targets. Clin Sci. 1999;96:513-23.

6. Tarjus A, Amador C, Michea L, Jaisser F. Vascular mineralocorticoid receptor and blood pressure regulation. Curr Opin Pharmacol. 2015;21:138-44.

7. Mineur YS, Belzung C, Crusio WE. Effects of unpredictable chronic mild stress on anxiety and depression-like behavior in mice. Behav Brain Res. 2006;175:43-50.

8. Chiba S, Numakawa T, Ninomiya M, Richards MC, Wakabayashi C, Kunugi $H$. Chronic restraint stress causes anxiety- and depression-like behaviors, downregulates glucocorticoid receptor expression, and attenuates glutamate release induced by brain-derived neurotrophic factor in the prefrontal cortex. Prog Neuro-Psychopharmacol Biol Psychiatry. 2012;39:112-9.

9. Wolkowitz O, Sutton M, Koulu M, Labarca R, Wilkinson L, Doran A, Hauger R, Pickar D, Crawley J. Chronic corticosterone Administration in Rats behavioral and biochemical-evidence of increased central dopaminergic activity. Eur J Pharmacol. 1986;122:329-38.

10. Yun J, Koike H, Ibi D, Toth E, Mizoguchi H, Nitta A, Yoneyama M, Ogita $K$, Yoneda $Y$, Nabeshima $T$, et al. Chronic restraint stress impairs neurogenesis and hippocampus-dependent fear memory in mice: possible involvement of a brain-specific transcription factor Npas4 J Neurochem. 2010;114:1840-51.

11. Huang PC, Li C, Fu TL, Zhao D, Yi Z, Lu Q, Guo LJ, Xu XL. Flupirtine attenuates chronic restraint stress-induced cognitive deficits and hippocampal apoptosis in male mice. Behav Brain Res. 2015;288:1-10.

12. Smith MA. Hippocampal vulnerability to stress and aging: possible role of neurotrophic factors. Behav Brain Res. 1996;78:25-36.

13. Uno H, Tarara R, Else JG, Suleman MA, Sapolsky RM. Hippocampal damage associated with prolonged and fatal stress in primates. J Neurosci. 1989:9:1705-11

14. Egeland M, Zunszain PA, Pariante CM. Molecular mechanisms in the regulation of adult neurogenesis during stress. Nat Rev Neurosci. 2015;16:189-200.

15. Kim JJ, Diamond DM. The stressed hippocampus, synaptic plasticity and lost memories. Nat Rev Neurosci. 2002;3:453-62.
16. Ryu JR, Hong CJ, Kim JY, Kim EK, Sun W, Yu SW. Control of adult neurogenesis by programmed cell death in the mammalian brain. Mol Brain. 2016;9:43.

17. Li WZ, Li WP, Yao YY, Zhang W, Yin YY, Wu GC, Gong HL. Glucocorticoids increase impairments in learning and memory due to elevated amyloid precursor protein expression and neuronal apoptosis in 12-month old mice. Eur J Pharmacol. 2010;628:108-15.

18. Sapolsky RM, Krey LC, Mcewen BS. Prolonged glucocorticoid exposure reduces hippocampal neuron number - implications for aging. J Neurosci 1985;5:1222-1227.

19. Yu IT, Lee SH, Lee YS, Son H. Differential effects of corticosterone and dexamethasone on hippocampal neurogenesis in vitro. Biochem Biophys Res Commun. 2004:317:484-90.

20. Schechter R, Whitmire J, Holtzclaw L, George M, Harlow R, Devaskar SU. Developmental regulation of insulin in the mammalian central-nervoussystem. Brain Res. 1992;582:27-37.

21. De Felice FG, Benedict CA. Key role of insulin receptors in memory, Diabetes. 2015;64:3653-5.

22. Biessels GJ, Reagan LP. Hippocampal insulin resistance and cognitive dysfunction. Nat Rev Neurosci. 2015;16:660-71.

23. Duarte Al, Moreira PI, Oliveira CR. Insulin in central nervous system: more than just a peripheral hormone. J Aging Res 2012;2012:384017.

24. Derakhshan F, Toth C. Insulin and the brain. Curr Diabetes Rev. 2013;9:102-16.

25. Zhao WQ, Alkon DL. Role of insulin and insulin receptor in learning and memory. Mol Cell Endocrinol. 2001;177:125-34.

26. Kleinridders A, Cai WK, Cappellucci L, Ghazarian A, Collins WR, Vienberg SG, Pothos EN, Kahn CR. Insulin resistance in brain alters dopamine turnover and causes behavioral disorders. Proc Natl Acad Sci U S A. 2015;112:3463-8.

27. Grillo CA, Piroli GG, Lawrence RC, Wrighten SA, Green AJ, Wilson SP, Sakai RR, Kelly SJ, Wilson MA, Mott DD, Reagan LP. Hippocampal insulin resistance impairs spatial learning and synaptic plasticity. Diabetes. 2015;64:3927-36.

28. Wang F, Song YF, Yin J, Liu ZH, Mo XD, Wang DG, Gao LP, Jing YH. Spatial memory impairment is associated with hippocampal insulin signals in Ovariectomized rats. PLoS One. 2014;9:e104450.

29. McNay EC, Ong CT, McCrimmon RJ, Cresswell J, Bogan JS, Sherwin RS. Hippocampal memory processes are modulated by insulin and high-fatinduced insulin resistance. Neurobiol Learn Mem. 2010:93:546-53.

30. Hanke S, Mann M. The Phosphotyrosine Interactome of the insulin receptor family and its substrates IRS-1 and IRS-2. Mol Cell Proteomics. 2009;8:519-34.

31. Bevan P. Insulin signalling. J Cell Sci. 2001;114:1429-30.

32. Van Obberghen E, Baron V, Delahaye L, Emanuelli B, Filippa N, GiorgettiPeraldi S, Lebrun P, Mothe-Satney I, Peraldi P, Rocchi S, et al. Surfing the insulin signaling web. Eur J Clin Investig. 2001;31:966-77.

33. Kim YC, Guan KL. mTOR: a pharmacologic target for autophagy regulation. J Clin Invest. 2015;125:25-32.

34. Marks DR, Tucker K, Cavallin MA, Mast TG, Fadool DA. Awake intranasal insulin delivery modifies protein complexes and alters memory, anxiety, and olfactory behaviors. J Neurosci. 2009;29:6734-51.

35. Zhang YL, Dai CL, Chen YX, lqbal K, Liu F, Gong CX. Intranasal insulin prevents anesthesia-induced spatial learning and memory deficit in mice. Sci Rep. 2016;6:21186.

36. Benedict C, Hallschmid M, Hatke A, Schultes B, Fehm HL. Born J, Kern W. Intranasal insulin improves memory in humans. Psychoneuroendocrinology. 2004;29:1326-34.

37. Benedict C, Hallschmid M, Schultes B, Born J, Kern W. Intranasal insulin to improve memory function in humans. Neuroendocrinology. 2007:86:136-42.

38. Stranahan AM, Arumugam TV, Cutler RG, Lee K, Egan JM, Mattson MP. Diabetes impairs hippocampal function through glucocorticoid-mediated effects on new and mature neurons. Nat Neurosci. 2008;11:309-17.

39. van Donkelaar EL, Vaessen KRD, Pawluski JL, Sierksma AS, Blokland A, Canete R, Steinbusch HWM. Long-term corticosterone exposure decreases insulin sensitivity and induces depressive-like behaviour in the $\mathrm{C} 57 \mathrm{BL} / 6 \mathrm{NCrl}$ mouse. PLoS One. 2014;9:e106960.

40. Osmanovic J, Plaschke K, Salkovic-Petrisic M, Grunblatt E, Riederer P, Hoyer S. Chronic exogenous corticosterone administration generates an insulinresistant brain state in rats. Stress-the International Journal on the Biology of Stress. 2010;13:123-31.

41. Moosavi M, Naghdi N, Maghsoudi N, Asl SZ. Insulin protects against stressinduced impairments in water maze performance. Behav Brain Res. 2007;176:230-6. 
42. Liu BJ, Zhang HY, Xu CQ, Yang GA, Tao JA, Huang JH, Wu JF, Duan XH, Cao $Y X$, Dong JC. Neuroprotective effects of icariin on corticosterone-induced apoptosis in primary cultured rat hippocampal neurons. Brain Res. 2011;1375:59-67.

43. Shintani T, Klionsky DJ. Autophagy in health and disease: a double-edged sword. Science. 2004;306:990-5.

44. Chung KM, Yu SW. Interplay between autophagy and programmed cell death in mammalian neural stem cells. BMB Rep. 2013;46:383-90.

45. Baehrecke EH. Autophagy: dual roles in life and death? Nat Rev Mol Cell Biol. 2005;6:505-10.

46. Gozuacik D, Kimchi A. Autophagy as a cell death and tumor suppressor mechanism. Oncogene. 2004;23:2891-906.

47. Chung KM, Park H, Jung S, Ha S, Yoo SJ, Woo H, Lee HJ, Kim SW, Kim EK, Moon C. Yu SW. Calpain determines the propensity of adult hippocampal neural stem cells to Autophagic cell death following insulin withdrawal. Stem Cells. 2015;33:3052-64.

48. Yu SW, Baek SH, Brennan RT, Bradley CJ, Park SK, Lee YS, Jun EJ, Lookingland KJ, Kim EK, Lee $\mathrm{H}$, et al. Autophagic death of adult hippocampal neural stem cells following insulin withdrawal. Stem Cells. 2008;26:2602-10.

49. Shen HM, Codogno P. Autophagic cell death: loch ness monster or endangered species? Autophagy. 2011;7:457-65.

50. Denton D, Nicolson S, Kumar S. Cell death by autophagy: facts and apparent artefacts. Cell Death Differ. 2012;19:87-95.

51. Kabeya Y, Mizushima N, Uero T, Yamamoto A, Kirisako T, Noda T, Kominami E, Ohsumi Y, Yoshimori T. LC3, a mammalian homologue of yeast Apg8p, is localized in autophagosome membranes after processing. EMBO J. 2000;19:5720-8.

52. Mizushima N, Yoshimori T. How to interpret LC3 immunoblotting. Autophagy. 2007;3:542-5.

53. Tanida I, Ueno T, Kominami E. LC3 and autophagy. Methods Mol Biol. 2008:445:77-88.

54. Ha S, Jeong SH, Yi K, Chung KM, Hong CJ, Kim SW, Kim EK, Yu SW. Phosphorylation of p62 by AMP-activated protein kinase mediates autophagic cell death in adult hippocampal neural stem cells. J Biol Chem. 2017;292:13795-808.

55. Paxinos G, Franklin KBJ: The mouse brain in stereotaxic coordinates. Compact 2nd EDN Amsterdam; Boston: Elsevier Academic Press; 2004.

56. Yeo BK, Hong CJ, Chung KM, Woo H, Kim K, Jung S, Kim EK, Yu SW. Valosincontaining protein is a key mediator between autophagic cell death and apoptosis in adult hippocampal neural stem cells following insulin withdrawal. Molecular Brain. 2016;9:31.

57. Nicholson DW, Ali A, Thornberry NA, Vaillancourt JP, Ding CK, Gallant M, Gareau Y, Griffin PR, Labelle M, Lazebnik YA, et al. Identification and inhibition of the ICE/CED-3 protease necessary for mammalian apoptosis. Nature. 1995;376:37-43.

58. Baek SH, Kim EK, Goudreau JL, Lookingland K, Kim SW, Yu SW. Insulin withdrawal-induced cell death in adult hippocampal neural stem cells as a model of autophagic cell death. Autophagy. 2009;5:277-9.

59. Yamamoto A, Tagawa $Y$, Yoshimori T, Moriyama Y, Masaki R, Tashiro Y. Bafilomycin a(1) prevents maturation of autophagic vacuoles by inhibiting fusion between autophagosomes and lysosomes in rat hepatoma cell line, H-4-II-E cells. Cell Struct Funct. 1998;23:33-42.

60. Ha S, Ryu HY, Chung KM, Baek SH, Kim EK, Yu SW. Regulation of autophagic cell death by glycogen synthase kinase-3 beta in adult hippocampal neural stem cells following insulin withdrawal. Molecular Brain. 2015;8:30.

61. Deacon RMJ. Assessing nest building in mice. Nat Protoc. 2006;1:1117-9.

62. Conrad CD, Galea LAM, Kuroda Y, McEwen BS. Chronic stress impairs rat spatial memory on the $Y$ maze, and this effect is blocked by tianeptine pretreatment. Behav Neurosci 1996;110:1321-1334.

63. Wright RL, Conrad CD. Chronic stress leaves novelty-seeking behavior intact while impairing spatial recognition memory in the Y-maze. Stress-the International Journal on the Biology of Stress. 2005;8:151-4

64. Kurek A, Kucharczyk M, Detka J, Slusarczyk J, Trojan E, Glombik K, Bojarski B, Ludwikowska A, Lason W, Budziszewska B. Pro-apoptotic action of corticosterone in hippocampal Organotypic cultures. Neurotox Res. 2016:30:225-38.

65. Crochemore C, Lu J, Wu Y, Liposits Z, Sousa N, Holsboer F, Almeida OFX Direct targeting of hippocampal neurons for apoptosis by glucocorticoids is reversible by mineralocorticoid receptor activation. Mol Psychiatry. 2005;10:790-8.
66. Heine VM, Maslam S, Zareno J, Joels M, Lucassen PJ. Suppressed proliferation and apoptotic changes in the rat dentate gyrus after acute and chronic stress are reversible. Eur J Neurosci. 2004;19:131-44.

67. Tishkina AO, Levshina IP, Lazareva NA, Passikova NV, Stepanichev MY, Ajrapetyanz MG, Gulyaeva NV. Chronic stress induces nonapoptotic neuronal death in the rat hippocampus. Dokl Biol Sci. 2009;428:403-6.

68. Lee T, Jarome T, Li SJ, Kim JJ, Helmstetter FJ. Chronic stress selectively reduces hippocampal volume in rats: a longitudinal magnetic resonance imaging study. Neuroreport. 2009;20:1554-8.

69. Copps KD, White MF. Regulation of insulin sensitivity by serine/threonine phosphorylation of insulin receptor substrate proteins IRS1 and IRS2. Diabetologia. 2012;55:2565-82.

70. Li L, Li XH, Zhou WJ, Messina JL. Acute psychological stress results in the rapid development of insulin resistance. J Endocrinol. 2013;217:175-84.

\section{Ready to submit your research? Choose BMC and benefit from:}

- fast, convenient online submission

- thorough peer review by experienced researchers in your field

- rapid publication on acceptance

- support for research data, including large and complex data types

- gold Open Access which fosters wider collaboration and increased citations

- maximum visibility for your research: over $100 \mathrm{M}$ website views per year

At BMC, research is always in progress.

Learn more biomedcentral.com/submissions 